\title{
Lung Myoepithelial Tumor
}

National Cancer Institute

\section{Source}

National Cancer Institute. Lung Myoepithelial Tumor. NCI Thesaurus. Code C142828.

A very rare, benign or malignant lung tumor with myoepithelial differentiation. 